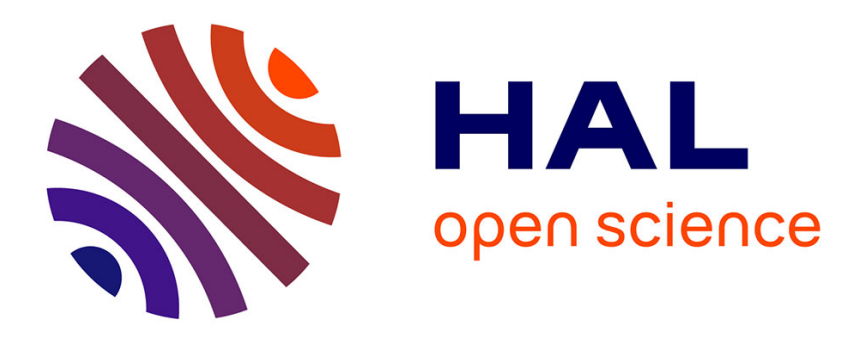

\title{
Une solution généralisée de l'équation de Poisson
}

Z.T. Kuznicki

\section{To cite this version:}

Z.T. Kuznicki. Une solution généralisée de l'équation de Poisson. Revue de Physique Appliquée, 1988, 23 (7), pp.1313-1316. 10.1051/rphysap:019880023070131300 . jpa-00245946

\section{HAL Id: jpa-00245946 https://hal.science/jpa-00245946}

Submitted on 1 Jan 1988

HAL is a multi-disciplinary open access archive for the deposit and dissemination of scientific research documents, whether they are published or not. The documents may come from teaching and research institutions in France or abroad, or from public or private research centers.
L'archive ouverte pluridisciplinaire HAL, est destinée au dépôt et à la diffusion de documents scientifiques de niveau recherche, publiés ou non, émanant des établissements d'enseignement et de recherche français ou étrangers, des laboratoires publics ou privés. 
Classification

Physics Abstracts

$73.30-73.40-73.40 \mathrm{~L}-73.40 \mathrm{Q}-73.40 \mathrm{~S}$

\title{
Une solution généralisée de l'équation de Poisson
}

\author{
Z. T. Kuznicki \\ Laboratoire d'Automatique et d'Analyse des Systèmes du CNRS, 7 avenue du Colonel Roche, 31077 Toulouse \\ Cedex, France
}

(Reçu le 17 décembre 1987, révisé le 11 février 1988, accepté le 24 mars 1988)

\begin{abstract}
Résumé. - Grâce à une représentation analytique combinée avec des procédures numériques appropriées, nous avons pu développer une solution approximative généralisée de l'équation de Poisson. Ayant une allure relativement très simple ainsi qu'une exactitude très poussée, cette solution met en évidence la composition macroscopique de la charge d'espace constituée de porteurs libres.
\end{abstract}

\begin{abstract}
Development of a generalized approximate solution of Poisson's equation based on an analytical representation combined with adapted numerical procedure is presented. This solution, having a relatively simple behaviour as well as a very advanced exactness, shows the macroscopic structure of the free carrier space-charge.
\end{abstract}

\section{Introduction.}

L'équation de Poisson :

$$
\varepsilon \varepsilon_{0} \operatorname{div} E=\rho
$$

où $\varepsilon, \varepsilon_{0}$ sont respectivement la permittivité du semiconducteur et du vide, relie la divergence de champ électrique $E$ à la densité de la charge d'espace $\rho$ (somme algébrique des charges fixes et mobiles).

Depuis longtemps, on connaît la première intégrale rigoureuse de l'équation (1), appelée équation Poisson-Boltzmann où au lieu de $\rho$ on met la répartition de concentration de la charge mobile en fonction de potentiel (statistique Maxwell-Boltzmann) [1-2].

\section{Solution rigoureuse.}

Grâce à cette solution, on découvre une longueur caractéristique, à savoir le rayon de Debye extrinsèque (devenu un paramètre du matériau) ainsi qu'une relation rigoureuse entre le champ électrique et le potentiel macroscopique [3-6]

où

$$
E(x)=\frac{k T}{q} \frac{\sqrt{2}}{L_{\mathrm{D}}} \sqrt{\exp [U(x)]-U(x)-1}
$$

$$
L_{\mathrm{D}}=\sqrt{\frac{\varepsilon \varepsilon_{0} \kappa T}{q^{2}} \frac{1}{N_{\mathrm{D}}}}
$$

est le rayon de Debye extrinsèque.
Quelques transformations de plus permettent de développer la loi électrostatique d'interfaces non perturbées [7] qui fournit des conditions aux limites exactes [8].

\section{Solution approximative.}

La seconde opération d'intégration de (1) ne peut s'effectuer (méthodes numériques exclues) qu'approximativement. Les solutions approximatives relativement simples n'apparaissent que dans deux cas extrêmes, à savoir très faibles ou très fortes perturbations de la neutralité électrique [6,9].

Pour de très faibles perturbations, la solution fait apparaître une seule longueur caractéristique, le rayon de Debye extrinsèque. Ce qui conduit à une répartition spatiale de potentiel normalisé donnée par une fonction exponentielle

$$
U(x)=U_{\mathrm{s}} \exp \left(-x / L_{\mathrm{D}}\right)
$$

où $U_{\mathrm{s}}$ est le potentiel normalisé à l'interface.

La solution équivalente pour de très fortes perturbations prend l'allure d'une fonction logarithmique [9] :

$$
U(x)=2 \ln \left[\exp \left(1 / 2 U_{0}\right)+x / L_{\mathrm{F}}\right]
$$

où

$L_{\mathrm{F}}$ est la longueur caractéristique de répartition de potentiel seulement près de l'interface, $0 \leqslant x<$ 
$\sqrt{2}\left(1-\sqrt{N_{\mathrm{D}} / n_{\mathrm{s}}}\right) L_{\mathrm{D}}$, souvent appelée : rayon d'écrantage,

$U_{0}$ est un potentiel réduit à l'interface,

$n_{\mathrm{s}}$ est une concentration de porteurs libres à l'interface.

Ainsi donc, la charge d'espace des porteurs libres contient deux zones d'influence : i) une zone peu perturbée avec une prépondérance de propriétés électriques du cristal occupé par cette charge et l'autre ii) fort perturbée avec une prépondérance de propriétés électriques au voisinage (jonctions) ou imposées de l'extérieur (polarisation).

\section{Solution généralisée.}

Nous avons alors admis une hypothèse, que la solution approchée généralisée (prenant en compte toutes les perturbations envisageables) doit respecter plusieurs longueurs caractéristiques, permettant le passage régulier entre faibles et fortes perturbations de neutralité électrique. De plus, pour garder l'avantage de la simplicité, nous avons admis que chaque répartition spatiale partielle est du type exponentiel (analogie à l'équation (4)). Ce qui donne une répartition spatiale type somme algébrique de $m$ termes

$$
U(x)=\sum_{j}^{m} U_{\mathrm{sj}} \exp \left(-x / L_{j}\right)
$$

où $m=1, \ldots, k ; k$-nombre naturel pas trop élevé, $U_{s j}$ est une composante de potentiel à l'interface, $L_{j}$ est la $j$-ième longueur caractéristique.

Le nombre $m$ dans l'équation (6) a été déterminé grâce au raisonnement suivant.

Côté mathématique: sachant que la répartition spatiale du potentiel macroscopique ne perd jamais son allure monotone et lisse, il est logique d'attendre que le nombre $m$ des termes de la somme (6) augmente avec l'exactitude d'approche représentée par (6), en commençant par 1 dans la limite des perturbations les plus faibles [6, 9].

Côté physique : les longueurs $L_{j}$ ayant des interprétations physiques ne peuvent pas avoir des valeurs inférieures aux distances atomiques.

Pour déterminer le nombre $m$ ainsi que les paramètres $U_{\mathrm{s} j}$ et $L_{j}$ (la notion de potentiel et de longueur peuvent être attribuées après une interprétation physique adéquate), nous avons recouru à une solution numérique d'un système de $2 m-1$ équations non linéaires.

Le système ainsi envisagé est composé de 3 équations exactes prenant en compte les valeurs exactes des potentiels, champ électrique et la concentration de porteurs libres (loi électrostatique) à l'interface [7] ainsi que $2(m-2)$ équations approchées supplémentaires reliant (en tout point à l'inté- rieur de la charge d'espace) le champ électrique calculé grâce à la formule exacte (2) au champ électrique obtenu grâce à la formule approchée (dérivé de (6)).

Dans la formule exacte (2) on n'a malheureusement que le potentiel approché. Comme points d'ajustement, nous avons choisi tout d'abord les points correspondants aux distances $L_{j}$.

Le choix des points d'ajustement dans notre procédure, pouvant être soumis à une optimisation numérique, a permis d'obtenir une exactitude relative telle qu'on a décidé d'abandonner une quelconque optimisation.

L'erreur relative est calculée à l'intérieur de notre procédure ainsi que par comparaison avec des solutions obtenues grâce aux méthodes numériques auto-compatibles. Elle ne dépasse jamais (dans toute la gamme de perturbations réellement envisageables) l'ordre de grandeur de $10^{-2} \quad\left(F_{\mathrm{s}} \leqslant 100\right)$ descendant même en dessous de $10^{-4} / 10^{-5}$ pour les perturbations moyennes où le champ électrique normalisé à l'interface $F_{\mathrm{s}}$ est inférieur à 10 .

Pour les perturbations intermédiaires, permettant de relier les deux cas extrêmes traités auparavant, on a fixé alors l'exactitude relative à $10^{-3}$, ce qui a permis d'obtenir le nombre $m=5$. Fixant une longueur au rayon de Debye extrinsèque, on constate que deux autres longueurs correspondent directement aux deux longueurs de bidimensionalisation du gaz de porteurs libres connues depuis longtemps dans la théorie de puits quantiques triangulaires [1012]. Les deux longueurs restantes caractérisent la perturbation moyenne et donnent l'épaisseur relative de la charge d'espace d'une structure abrupte $n^{+}-n\left(p^{+}-p\right)$ exprimée dans la loi électrostatique [7].

\section{Interprétation.}

Le potentiel électrostatique macroscopique (vu de l'extérieur de la charge d'espace), nommé habituellement potentiel de diffusion, étant imposé par la loi électrostatique des homo-structures semiconductrices abruptes [7], peut être représenté comme la résultante des potentiels partiels provenant des $m$ fractions de porteurs constituant la charge d'espace (Eq. (6)).

Cela implique que le nombre total $N$ de porteurs libres se partage à l'intérieur de la charge d'espace en quelques $m$ fractions thermalisées différemment [14].

La thermalisation amène à l'agitation isotropique des porteurs d'équilibre et à $m-1$ types d'agitation anisotropique des porteurs excédentaires. Ce phénomène au niveau microscopique est dû à l'opposition des forces de diffusion (pression du gaz de porteurs libres) d'un côté et des forces électrostatiques provoquées par cette même diffusion de l'autre côté.

Un exemple de nos résultats est donné sur les 
figures 1-5 où on les compare aux données publiées auparavant par les autres auteurs [10-12].

$\mathrm{La}$ figure 1 représente en effet trois des plus grandes longueurs caractéristiques $L_{j}$ (type rayon de Debye), dont le rayon de Debye extrinsèque $L_{\mathrm{D}}$, tandis que la figure 2 - donne les deux plus petites longueurs caractéristiques $L_{\mathrm{E}}$ et $L_{\mathrm{G}}$ en fonction de la perturbation de neutralité électrique (caractérisée par le champ électrique normalisé $\left.F_{\mathrm{s}}\right)$. A titre de comparaison on a représenté dans la figure 1 deux longueurs supplémentaires : le rayon d'écrantage $L_{\mathrm{F}}$ (distance au bout de laquelle la densité de la charge d'espace diminue $e$-fois) ainsi que l'épaisseur de la charge d'espace $L_{\mathrm{B}}$ [13].

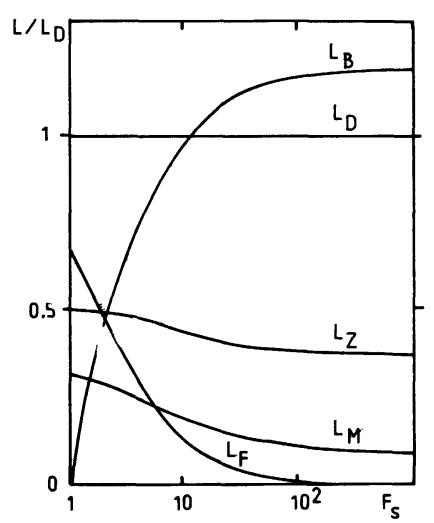

Fig. 1. - Représentation des longueurs caractéristiques normalisées $L_{\mathrm{M}}, L_{\mathrm{Z}}$ (correspondant à la loi électrostatique) en fonction du champ électrique normalisé à l'interface, $F_{\mathrm{s}}$. $L_{\mathrm{B}}$ l'épaisseur de la charge d'espace [13], $L_{\mathrm{F}}$ le rayon d'écrantage. A titre de comparaison nous avons porté $L_{\mathrm{D}}$, rayon de Debye extrinsèque.

[Normalized caracteristic length $L_{\mathrm{M}}, L_{\mathrm{Z}}$ (related to the electrostatic law) as a function of normalized electric field on the interface $F_{\mathrm{s}} . L_{\mathrm{B}}$ space-charge depth [13] and $L_{\mathrm{F}}$ screening length. In comparison with the extrinsic Debye length $L_{\mathrm{D}}$ is shown.]

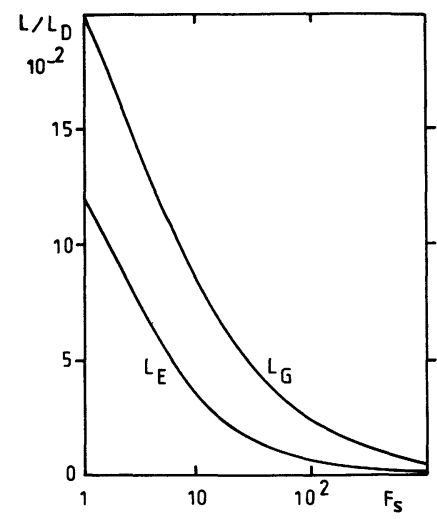

Fig. 2. - Variation des deux plus petites longueurs caractéristiques normalisées : $L_{\mathrm{E}}$ et $L_{\mathrm{G}}$ en fonction de la perturbation de neutralité électrique.

[Two shortest characteristic lengths $\left(L_{\mathrm{E}}\right.$ and $\left.L_{\mathrm{G}}\right)$ variation as a function of electric neutrality perturbation.]
Sur la figure 3 on compare $L_{\mathrm{G}}$ avec l'épaisseur de canal quantique à la surface d'un cristal semiconducteur telle qu'elle est définie par Stern [10] et reprise ensuite par Schwarz et Russek [12]. On remarque une bonne concordance pour une très large plage de valeurs du champ électrique.

Remarque : les longueurs $L_{\mathrm{E}}$ et $L_{\mathrm{G}}$ sont dans la plupart des cas beaucoup plus petites que les rayons de Debye extrinsèques respectifs, calculés sur la base de l'équation (3) avec les valeurs de concentration des porteurs excédentaires au voisinage de la surface compatibles.

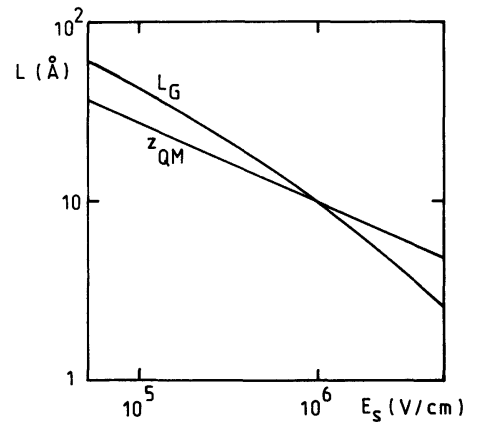

Fig. 3. - Comparaison de $L_{\mathrm{G}}$ avec l'épaisseur de canal quantique $Z_{\mathrm{QM}}$ [12] en fonction du champ électrique à l'interface.

[Comparaison of $L_{G}$ to the quantum-mechanical channel width $Z_{\mathrm{OM}}$ [12] as a function of electric field at the interface.]

La figure 4 représente les répartitions des populations dans les cinq fractions des porteurs libres en fonction de la perturbation (champ électrique normalisé $F_{\mathrm{s}}$ ). Cette situation pourrait avoir lieu par exemple dans une structure type MOS soumise à différentes polarisations de grille.

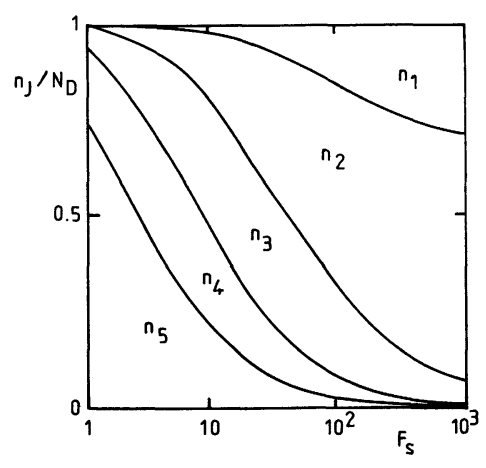

Fig. 4. - Répartition des fractions de porteurs libres correspondant aux longueurs caractéristiques $L_{j}$ d'une couche d'accumulation en fonction de perturbation.

[Free carrier fraction distributions related to accumulation layer characteristics lengths $L_{j}$ as a function of perturbation.] 
Sur la figure 5 on a porté la comparaison des répartitions de la somme de deux fractions des porteurs libres liées aux plus petites longueurs caractéristiques $L_{\mathrm{E}}$ et $L_{\mathrm{G}}$ (courbe nommée class.) avec les répartitions des fractions des porteurs libres de la plus basse bande d'énergie quantifiée dans le puits quantique triangulaire, rapportées par Stern pour trois orientations cristallographiques différentes de la surface du silicium $[10,11]$.

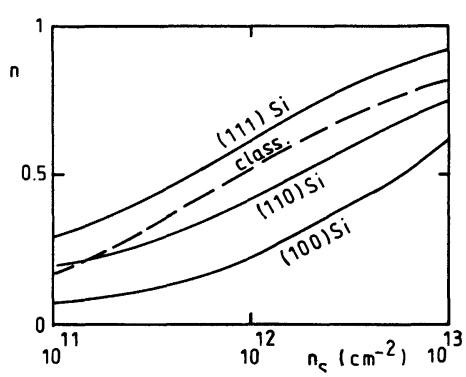

Fig. 5. - Comparaison des répartitions des deux fractions de porteurs libres liées aux longueurs caractéristiques: $L_{\mathrm{E}}$ et $L_{\mathrm{G}}$ avec les répartitions des fractions de porteurs libres de la plus basse bande d'énergie quantifiée dans le puits quantique $[10,11]$.

[Free carrier fraction distribution related to the characteristic lengths $L_{\mathrm{E}}$ and $L_{\mathrm{G}}$ compared to the free carrier fraction distribution in the lowest subband of the quantum well $[10,11]$.

Ainsi donc, sans entrer dans les détails on constate facilement qu'il y a une étonnante concordance des résultats obtenus précédemment, dans le cadre de la théorie quantique et des résultats dits classiques, rapportés dans ce travail. Outre la concordance des distances $L_{\mathrm{G}}$ et $z_{\mathrm{QM}}$ (Fig. 3) on a un bon accord des populations de fractions respectives des porteurs (Fig. 5).

Ce qui nous permet d'affirmer que ces liens, postulés déjà entre autre par Schwarz et Russek [12], existent effectivement.

\section{Conclusions.}

Prenant en compte le concept de refroidissement graduel de porteurs libres à l'intérieur d'une charge d'espace [14], la méthode que nous venons d'exposer est aussi applicable dans le cas de structures dégénérées où seules les conditions aux bords liées à la statistique Maxwell-Boltzmann doivent être remplacées par celles déterminées dans le cadre de la statistique Fermi-Dirac.

De plus, il est possible de compléter le concept de réchauffement électronique [15-16] résultant d'une polarisation extérieure appliquée au cristal contenant la charge d'espace.

Enfin, à partir de notre méthode, nous sommes capables de reproduire la caractéristique directe statique courant-tension des jonctions fortement dissymétriques, ne prenant en compte que les porteurs majoritaires. Ceci peut servir de référence de traitement des résultats expérimentaux concernant les structures réelles.

\section{Remerciements.}

Je tiens à remercier le Ministère des Affaires Etrangères et le CNRS pour le soutien financier qu'ils m'ont accordé. Je suis très reconnaissant au Professeur A. Martinez du LAAS pour avoir facilité ma venue en France ainsi que pour les fructueuses discussions.

Le traitement numérique a été développé en coopération avec Monsieur P. Gouyon du LAAS.

\section{Bibliographie}

[1] Garrett, C. G. B., Brattain, W. H., Phys. Rev. 99 (1955) 376.

[2] Kingston, R. M., Neustadter, S. I., J. Appl. Phys. 26 (1955) 718.

[3] Adirovich, E. I., Riabinkin, I. U., Temko, K. V., Sov. Phys. Tech. Phys. 3 (1958) 49.

[4] VAN DE Wiele, F., Demoulin, E., Solid State Electron. 13 (1970) 717.

[5] Kennedy, D. P., IEEE Trans. Electron. Devices ED22 (1975) 988.

[6] MANY, A., Surf. Sci., Lectures presented at International Course, Trieste Italy (16 Janv.-10 Avril 1974) Vienna, Austria, IAEA (1975) p. 447-500.

[7] KuZNICKI, Z. T., Thin Solid Films 85 (1981) 169.
[8] Kuznicki, Z. T., Bull. Acad. Polon. Sci. Ser. Sci. Tech. 29 (1981) 111.

[9] KUZNICKI, Z. T., Rapport du LAAS 87131 (mai 1987).

[10] Stern, F., Phys. Rev. B 5 (1972) 4891.

[11] Ando, T., Fowler, A. B., Stern, F., Rev. Mod. Phys. 542 (1982).

[12] Schwarz, S. A., RusseK, S. E., IEEE Trans. Electron. Devices 30 (1983) 1634.

[13] KuZnicki, Z. T., Zimmermann, B., Revue Phys. Appl., soumis à publication.

[14] KUZNICKI, Z. T., C.R.A.S., soumis à publication.

[15] GunN, J. B., J. Electron. 2 (1956) 87.

[16] Mendelson, K. S., Bray, R., Proc. Phys. Soc. B 70 (1957) 899. 